\title{
Urban road network matching method based on stable stroke hierarchical structure
}

\author{
Lin Yang ${ }^{1 *}$, Run Wang ${ }^{12}$, Han Yan ${ }^{12}$ \\ ${ }^{1}$ Faculty of Information Engineering, \\ China University of Geoscience(Wuhan), Wuhan,china \\ ${ }^{2}$ Graduate Department, China University of Geoscience(Wuhan), Wuhan,china \\ Corresponding author:gisyanglin@gmail.com
}

\begin{abstract}
Road matching plays an important role on road data integration. The geometric similarity matching often become invalid when the calculation is considered only in local level, this paper excavates stable calculation space from the urban spatial structure, and makes full consideration of stable hierarchical stroke structure to the control of road features. After that, the recognition ability to changed features is improved by the relationship calculation between stable neighborhood space and the road features. In this paper, the algorithm is improved based on global consistency matching method, and a stable stroke based on hierarchical structure of global matching algorithm is established. The method is verified on the selected road data through experiment, and the matching result is preferable.
\end{abstract}

Key words-road matching, stable stroke, hierarchical structure, probabilistic relaxation method.

\section{INTRODUCTION}

Facing with the rapid developing and changing of China's road infrastructure, the demand of latest electronic maps increases every year. Thus, an effective method of map matching can serve the map integration and map fusion, and effectively reduce the high cost of reduplication of data collection; it also can detect map data changes targets in order to achieve incremental update and improve the electronic map updates and current situation. It can be said that, the map matching is the basic technique to achieve change detection map data, maps integration, integration and updating ${ }^{[1]}$.

Early in the last century, some scholars began to study the map matching, a lot of vector spatial target matching method were proposed. However, as data acquisition technology progressing, road network data becomes more complex, multisource and multi-scale phase-reflected, which makes the road network matching algorithms facing more and more problems.

1) Weight set and mismatch.

Typically, the matching method requires a variety of metric indicators and combining them to achieve a more accurate matching result, these matching method usually used a direct measure of the absolute distance between targets (such as the distance difference, angle difference), to obtain an integrated average weighted metric value of the similarity. There are two problems in this way, On the one hand, the lack of theoretical support to set the value of the indicator weights and thresholds which are mostly rely on experience result in a match result unstable, and different weights will lead to different match results; On the other hand, the map data exits uneven deviation, this direct measure method is easy to mistake the near arc segment or point of identified to the same name and the same target, generating false match.

2)Complex matching conditions.

There are several matching conditions. Data acquisition and production in different ways, result in spatial object matching exists 1:0、1:1 $1: \mathrm{M}$ and $\mathrm{N}: \mathrm{M}$ and a variety of possible matches. Different data sources reflect the complexity of the different matching situations, then there must be matching algorithm designed to consider many possibilities for each matching. However, most algorithms start from the details, that is from the node or the segment of the reference map looking for a candidate or a match, to discuss the matching.

This paper analyzes the status of the road of the matching algorithm, presents a stable stroke hierarchical structure for urban road network matching method aimed at the above problem.

This structure can maintain the road connectivity, continuous data can be a very good performance for the road structure, which is help for search the stability of space elements; in addition, stroke as the matching unit, can be more easily from the overall road to find the road, and 1:1,1:M,and $\mathrm{N}$ :M matching from the data.

In addition, the algorithm in multi-disciplinary indicators draws on the experience of previous scholars, the probabilistic relaxation method is introduced matching algorithms[]. Probabilistic relaxation method can not only start from a global perspective select the best matching set, improve the matching accuracy but also integrate a variety of similarity metrics, reducing the impact of weight.

Finally, this article through experiments prove that this steady stroke hierarchical structure approach would both global features and local features, in overcoming local mismatch situation in the match and handle a variety of produce a good result. 


\section{STABLE STROKE HIERARCHICAL STRUCTURE}

Stable stroke is existed in the two matched road map, which could also be called the public stroke. The Public stoke designated as the coordinate axes in the coordinate system by which an "Axis" positioning other stroke location, and thus, even if there is non-uniform deformation of the map, name-like stroke also can be found based on the relative position between the stroke location and road element. Moreover, the urban road network has obvious hierarchy, so the road can be divided to expressway, trunk roads, secondary roads, as well as branches when designing the road. Although map data often lacks level semantics, the concept of hierarchy can still be perceived by people through stroke structure. Stable stroke hierarchical structure matching method is dependent on this level of the road network.

The algorithm uses a recursive idea. Firstly, on the highlevel of the road, extract the common stroke between original map and the to be matched maps, since the public strokes have been found by the corresponding relationship between the matching, that this part of the road can be considered as a stable structure, and be able to provide a lower matching reference; Then compute relative distance between the lower layer and the upper road according to the similarity measure of the model, and the shape of the stroke itself is similar scheme, the combined effects of internal nodes matching the road network; finally matched pairs of strokes and then added to the stable structure, followed by cycle stroke next level matching.

The algorithm has two steps (as Fig. 1).

1) Generate an initial public stroke reference. In this step, we extract strokes A 'and B' from two be matching maps A and $\mathrm{B}$, then matching them to obtain its public portion to serve as the initial common reference layer of the layered stroke.

2) Match in each stroke layer. Find an initial public stroke of the reference adjacent to form a new layer stroke, the relative distance of reference similarity index between the new road plan and the initial public to introduction of comprehensive metrics, by probabilistic relaxation iterative method to obtain the most best match set; then will determine the best match for adding to the initial public stroked reference, to form the new public reference. Continue to find new public stroke adjacent to draw reference to match. Repeat this step until there is no strokes can add up; then further define the path is not formed before the matching stage plan or arc segments were excluded to form the final match set.

\section{A. Generating an initial public stroke reference}

In order to ensure that the resulting initial public stroke reference matching accuracy and efficiency, to reconstruct the road data, generate stroke structure, and then propose selection methods through road network in high-grade road plan sets A 'and B'. High-grade roads can represent the basic shape of the road network, the amount of data is small, the road network plays an important role, are less likely to change, with a strong stability, and high-grade road distance between the large mismatch of relatively unlikely.

Assumptions were to be matched from the two road network map vector data A, and B extracted two sets - stable reference A 'with stable reference B', then this article using probabilistic relaxation method, and as stroke units the match extraction A 'and B' in the same part.

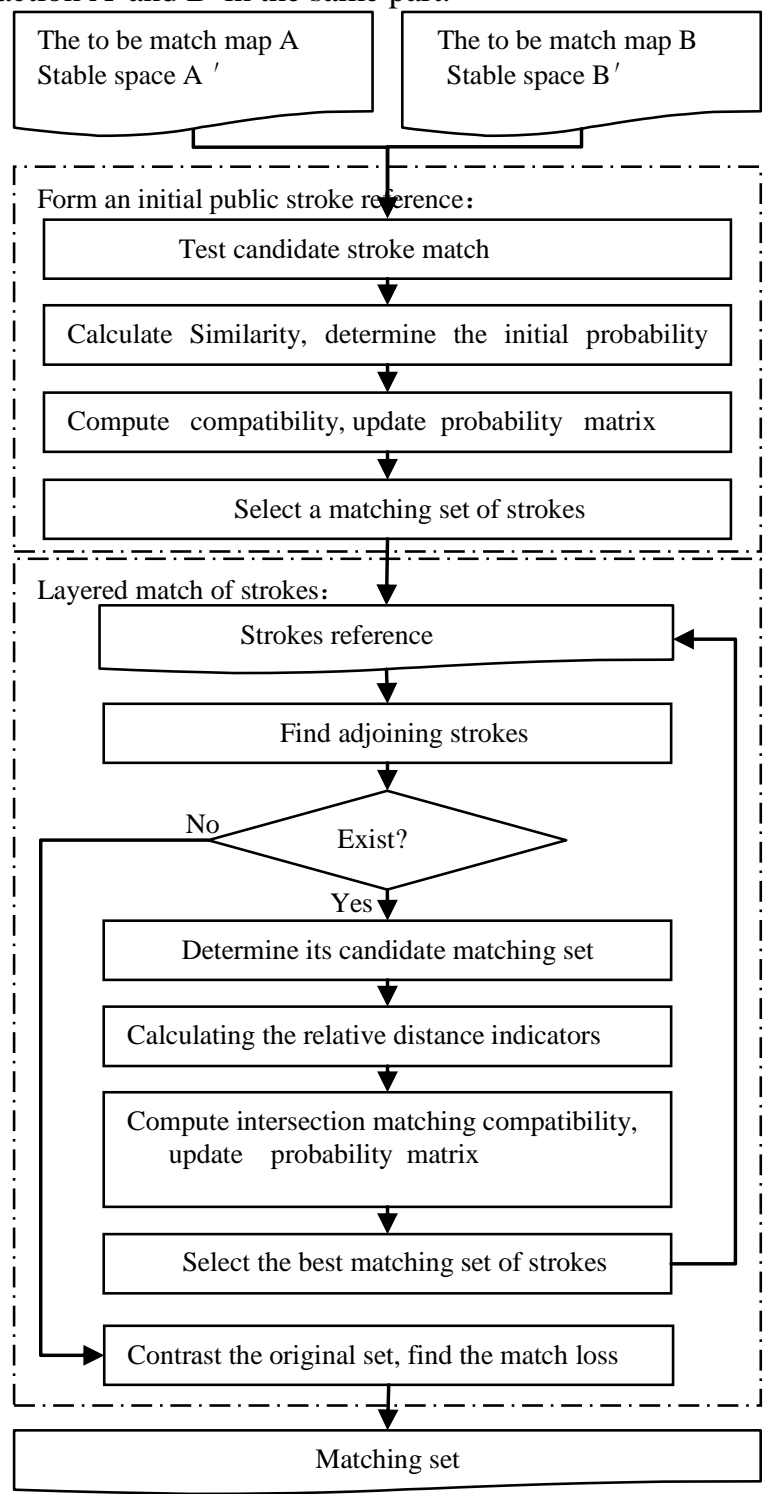

Fig.1. Matching Algorithmic Process

Based on a different match between point and arc, the match based on strokes is from coarse to fine matching process, draw the shape of the road coarse matching rate structure, through an internal node topology similarity exact matching. Determined in this way draw the stroke corresponding to the internal nodes determined simultaneously the matching of 1:1、1:M and N:M . Matching specific steps are as follows:

\section{1) Determine the initial probability}

Using of buffer analysis to determine $\mathrm{A}^{\prime}$ in $\mathrm{B}^{\prime}$ match in the candidate set $B_{C S} \subseteq B^{\prime}$. In a stable reference in every road draw at least one match road plan exists to form a stable reference, so if there is a situation of $\boldsymbol{B}_{C S}=\boldsymbol{\phi}$, that is $\boldsymbol{a}^{\prime}$ in 
which no candidate matching set, it will be excluded $a^{\prime}$ from the original stable reference. Let identified a road designated as a candidate match. Define the initial probability $\boldsymbol{P}_{a^{\prime} b^{\prime}}^{(0)}$ as $a^{\prime}$ each one with the $b^{\prime} \in B_{B C}$ matching degree. As shown in "Equation 1".

$$
P_{a^{\prime} b^{\prime}}^{(0)}=\frac{\operatorname{Sim}_{a^{\prime} b^{\prime}}}{\sum_{b^{\prime} \in B_{C S}} \operatorname{Sim}_{a^{\prime} b^{\prime}}}
$$

This matches by the linear similarity $\operatorname{Sim}_{\text {line }}$ and each node in the stroke the average topological similarity to measure Sim $_{\text {node }}$, as shown in "Equation 2".

$$
\operatorname{Sim}_{a^{\prime} b^{\prime}}=\operatorname{Sim}_{\text {line }} \cdot \operatorname{Sim}_{\text {node }}
$$

First mentioned in the literature[][] using the formula to calculate the line shape similarity $\operatorname{Sim}_{\text {line }}$.

$$
\left\{\begin{array}{l}
\operatorname{Sim}_{\text {line }}=\sum\left(s_{i+1}-s_{i}\right) \cdot a\left(V_{i}^{a^{\prime}}, V_{i}^{b^{\prime}}\right) \\
a\left(V_{i}^{a^{\prime}}, V_{i}^{b^{\prime}}\right)=\frac{\min \left\{\left|V_{i}^{a^{\prime}}\right|,\left|V_{i}^{b^{\prime}}\right|\right\}}{\max \left\{\left|V_{i}^{a^{\prime}}\right|,\left|V_{i}^{b^{\prime}}\right|\right\}} \cos \left(V_{i}^{a^{\prime}}, V_{i}^{b^{\prime}}\right)
\end{array}\right.
$$

Then calculate the internal node (endpoint, inflection, intersection) of average topological similarity Sim $_{\text {node }}$.

Before measure the node similarity, need to find the corresponding relationship between the node. The hausdorff distance between stroke $a^{\prime}$ and stroke $b^{\prime}$ is $D_{\text {hausdorff }}$, from one end of the $a^{\prime}$, calculated at each point of $a^{\prime}$ to every point of $b^{\prime}$ on the distance $d$, if $d<D_{\text {hausdorff }}$, then as the candidate matching points. The topological similarity of node $i$ can draw from its adjacent road similarity to describe, as shown in "Equation 4".

$$
\operatorname{Sim}_{\text {node }(i)}^{a ' b^{\prime}}=1 / n A E \sum a\left(e_{a}, e_{b}\right)
$$

$n A E$ (Adjacent Edges) is the number of adjacent edges other than their own; the vector of $e_{a}$ and $e_{b}$ representing the node to be matched, between ${ }^{a_{i}}$ and $b_{j}$, in addition to stroke itself adjoining the corresponding arc, arc similarity $a\left(e_{a}, e_{b}\right)$ calculated as shown in "Equation 3".

Since the internal node number of different in stroke $\boldsymbol{a}^{\prime}$ and $\boldsymbol{b}^{\prime}$, the matching possibilities are different,(a)When a candidate points have multiple candidate matching point, the need for further use of topological structural similarity to select, the paper selected large topology similar candidate points to determine the relationship between a 1:1 match.(b)If the point is not the candidate point such as point $a_{4}$, the corresponding topological similarity is zero.

2) Probability iterative update probability matrix

In order to determine the matching of the strokes, it is need to consider that to be matched strokes and the adjacent matching strokes that matches its support in the stability of space A 'and B'. Algorithm selected two indicators measure the degree of compatibility $t$, namely the angle $\theta$ between the adjacent strokes and the ratio $S$ of the distance between the intersection of the adjacent strokes and the starting stroke to the length of strokes. Using "Equation7" calculation of compatibility coefficient $C\left(a^{\prime}, b^{\prime} ; a^{\times}, b^{\times}\right), a^{\times}, b^{\times}$is the intersection strokes of $a^{\prime}, b^{\prime}$.

$$
\begin{aligned}
& C\left(a^{\prime}, b^{\prime} ; a^{\times}, b^{\times}\right)= \\
& \quad \sum \frac{1}{2\left(1+\left(t_{a^{\prime} a^{\times}}-t_{b^{\prime} b^{\prime}}\right)^{2}\right)} t=\{\theta, S\}
\end{aligned}
$$

To ensure compatibility degree satisfy symmetry requirements,

$$
\begin{aligned}
& C\left(a^{\prime}, b^{\prime} ; a^{\times}, b^{\times}\right)=C\left(a^{\times}, b^{\times} ; a^{\prime}, b^{\prime}\right) \\
& =\sqrt{C\left(a^{\prime}, b^{\prime} ; a^{\times}, b^{\times}\right) \cdot C\left(a^{\times}, b^{\times} ; a^{\prime}, b^{\prime}\right)}
\end{aligned}
$$

In the r-th iteration, the adjacent of strokes support is as following:

$$
S_{a^{\prime} b^{\prime}}^{(r)}=\frac{1}{m-1} \sum_{a^{\prime} \in A^{\prime}, b^{\prime} \in B_{C S}} \underset{\operatorname{ax}}{ }\left[C\left(a^{\prime}, b^{\prime} ; a^{\times}, b^{\times}\right) P_{a^{\times} b^{\times}}^{(r)}\right]
$$

Using “Equation 8" updated probability matrix.

$$
P_{a^{\prime} b^{\prime}}{ }^{(\mathrm{r}+1)}=\frac{p_{a^{\prime} b^{\prime}}{ }^{(r)}+q_{a^{\prime} b^{\prime}{ }^{(r)}}}{1+\sum_{b^{\prime} \in B_{C S}} q_{a^{\prime} b^{\prime}}{ }^{(r)}}
$$

When the iterative changes less than the threshold, then stop the calculation.

\section{3) Matching set selection}

In the final probability matrix $\boldsymbol{P}^{(r)}$, select the maximum value in the matrix, namely the most likely match, and the column and the row is removed from the probability matrix, repeat this step until no value can be selected. Elected matching relationship as initially public stroke reference, 
namely $\mathrm{Ora}_{A}$ and $\mathrm{Ora} \boldsymbol{B}_{\text {, strokes in the }} \mathrm{Ora} \boldsymbol{A}_{A}$ and strokes in the $\mathrm{Ora}_{B}$ are matching each other .

\section{B. Strokes layered match}

Initial public reference $O r a_{A}$ and $O r a_{B}$ are ensure to the next stroke level. After determining the match result, it will add the success match of the strokes to the initial public strokes, form of a new layer of reference to the public strokes, use of recursive thinking, the next level strokes will be find to match again .

\section{EXPERIMENTS AND CONCLUSIONS}

This paper selects the city of Wuhan in 2005 and 2011 of city road map as the experimental data. Firstly extract the skeleton, then analysis skeleton of line similarity for 2005 and 2011, and finally concluded that in 2005 the city has 92 percent of the skeleton line stability with respect to the 2011 urban skeleton. Through the analysis on the stability of the road, indicating that city's main thoroughfares, major roads do have good stability.

Using this method for matching two different sources of Wuhan road, the original layer has 724 roads and 1061 nodes, the objective layer has 768 roads and 1082 nodes. According to the experiment, the matching rate is $97.6 \%$, and the accuracy is $96.5 \%$, the matching process cost 52 seconds. The accuracy and efficiency of the algorithm is ideal.

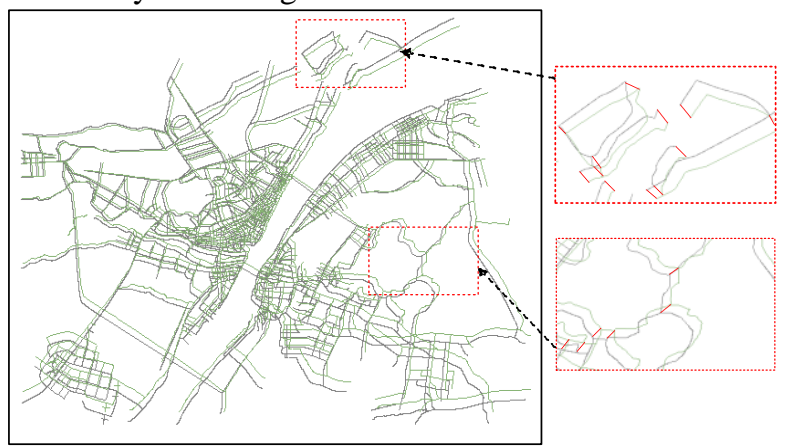

Fig.2. Matching effect of Wuhan road

The author believes that high-grade road represents the basic shape of the road network, the roads network play an important role, are less likely to change, with a strong stability, and the large distance between high-grade road, the possibility of mismatching relatively small. This proved by experiments that stable stroke based on hierarchical structure approach would have both global features and local features, it is make a good result for overcoming local mismatch situation in the match and handle a variety of match situations.

\section{ACKNOWLEDGMENT}

This work has been supported by the National Natural Science Foundation of China (Project No. 41201385).

\section{REFERENCES}

[1] S.Mustière, T.Devogele, "Matching Networks with Different Levels of Detail" Geoinformatica (2008) 12., pp.435-453.

[2] J.J.Ruiz, F.J.Ariza, M.A. Ureña, E.B. Bla’zquez "Digital map conflation: a review of the process and a proposal for classification," International Journal of Geographical Information Science, Vol. 25, September 2011,pp. 1439-1466.

[3] W.Song, J.M.Keller, T.L.Haithcoat, C.H.Davis, "RelaxationBased Point Feature Matching for Vector Map Conflation," Transactions in GIS, 2011, pp.43-60.

[4] E.Safra, Y.Kanza, Y.Sagiv, C.Beeri and Y.Doytsher," Location-based algorithms for finding sets of corresponding objects over several geo-spatial data sets," International Journal of Geographical Information Science, January 2010,Vol.24,pp.69-106.

[5] B.Yang, Y.Zhang, X.Luan, "A probabilistic relaxation approach for matching road networks," International Journal of Geographical Information Science,2013,Vol.27,pp.319-338.

[6] Y.Huh, K.Yu, and J.Heo," Detecting conjugate-point pairs for map alignment between two polygon datasets," Computers, Environment and Urban Systems , 2011,pp. 250-262.

[7] D. Zhao, Y. Sheng, H.Guo," An algorithm for automatically matching corresponding points on homonymous map features," Proc. of SPIE Vol.pp. 7146 - 71461J-1.

[8] E. Safra, Y. Kanza, Y. Sagiv, Y. Doytsher," Ad hoc matching of vectorial road networks," International Journal of Geographical Information Science,2013,Vol.27,pp.114-153. 